\title{
Fairness-Oriented Link Scheduling for a D2D-enabled LTE-U/Wi-Fi Coexistence Network
}

\author{
Chigang XING, Fangmin LI*
}

\begin{abstract}
To avoid spectrum crunch and improve spectrum efficiency, the use of unlicensed spectra and the introduction of D2D communication will be areas of focus in communication development. However, in the existing unlicensed spectrum coexistence mechanism, different ways of communication are seen as hindering each other. In this paper, we deliberate the coexistence of a D2D-enabled LTE network with Wi-Fi under an unlicensed band. Unlike previous coexistence mechanisms, we allow cochannel transmission, and our goal is to make full use of the advantages of D2D proximity communication and achieve fairness in co-channel transmission. First, we modeled the coexistence network and derived the expressions coverage probability of all types of receivers. Based on the analytical model and simulation results, we prove that D2D communication can be exploited to achieve fairness requirements in co-channel transmission over the unlicensed band. We rephrase the fairness schedule problem as a mixed-integer nonlinear optimization problem for D2D density and transmit power, and we use an Ortho-MADS algorithm to solve it. The simulation results show that the proposed scheme can use D2D communication to improve the fairness of the system.
\end{abstract}

Keywords: D2D; fairness; resource allocation; unlicensed band; Wi-Fi

\section{INTRODUCTION}

The rapid growth of mobile traffic and user demand for a high quality of mobile service is approaching the limits of the current LTE system [1]. To cope with this development trend of mobile communications, researchers have adopted two main approaches. To increase network capacity, LTE is moving towards the utilization of the unlicensed band (LTE-U) by coexisting with other nonmobile communication systems, such as $\mathrm{WiFi}[2,3]$, and to enhance spectral efficiency, LTE is introducing more access models such as device-to-device (D2D) communication on LTE networks [4]

In unlicensed band, most of the researches focuses on LTE-U and Wi-Fi coexist. LTE-U and Wi-Fi operating in the same band will cause significant performance degradation $[5,6]$. To avoid degradation, Wi-Fi requires a coexistence mechanism for spectrum sharing. In the coexistence of mobile communication systems and nonmobile communication systems, research has taken two significant lines: (a) listen before talking [7, 8]; (b) dutycycle muting [9]. These two types of methods mainly avoid resource contention in the time domain. In addition to these two methods, interference coordination has also been considered for achieving coexistence. In [10], the authors proposed a coexistence scheme to guarantee the quality of service (QoS) by optimally distributing almost blank subframes (ABSs) over the frame. However, the MAC protocol cannot completely prevent Wi-Fi users from being affected by LTE-U transmitters. Therefore, 3GPP further put forward the requirement of fairness [11].

In further research $[12,13]$, the authors indicate that fairness is affected by the number of LTE-U base stations and its transmit power.

Since transmit power is an important factor affecting Wi-Fi users, researchers attempt to allocate the unlicensed spectrum to lower-power cellular communication methods, such as D2D communication [14]. The introduction of D2D will add additional sources of interference and affect both LTE and WiFi users $[15,16]$. In doing so, a coexistence mechanism is needed that allows D2D to operate in unlicensed bands with protective fairness measures for LTE-U and Wi-Fi transmissions.
To introduce the D2D communication method, the researchers studied from three directions: model selection [17], power control [17, 18], spectrum allocation $[18,19]$, and channel access mechanism $[20,21]$. Similar to the coexistence mechanism of LTE-U and Wi-Fi, researchers also hope to avoid interference in the time domain. In [22], the authors propose time-division scheduling (TDS) to minimize interference.

Similarly, in D2D-enabled coexistence scenarios, scholars have also explored the need for fairness. Reference [23] takes the Wi-Fi performance as a penalty term into the objective function when addressing fairness in a D2D-enabled unlicensed band coexistence scenario. They proposed a swap matching algorithm to allocate the unlicensed channel and maximize the total system throughput. When analyzing fairness, the penalty function method is used to substitute the Wi-Fi indicator into the objective function. Similarly, Reference [24] proposes a joint optimization algorithm for mode selection and resource allocation to resolve mode switching and spectrum sharing at the same time.

In the existing coexistence mechanism, due to the characteristics of mobile communications, transmitters in the cellular system such as D2D and eNB are considered obstacles to Wi-Fi communication. Under this premise, the ideal model is used when constructing the optimization problem. Therefore, the core of the coexistence mechanism has invariably been to avoid or reduce interference. However, due to the propagation characteristics of electromagnetic waves, collisions are unavoidable. So, in this paper, we focus on how to take advantage of the low power consumption characteristics of D2D communication to achieve fair coexistence when co-channel is unavoidable.

In this paper, we consider a D2D schedule scheme in a D2D-enabled unlicensed band coexistence scenario over the $5 \mathrm{GHz}$ unlicensed spectrum. We propose a D2D utilization scheme, which means that fairness between cellular users and non-cellular users can be achieved by introducing D2D communication. The main contributions of this paper are summarized as follows:

We present an analytic model based on stochastic geometry for the analysis of a D2D LTE and Wi-Fi 
coexistence scenario and derive the general average coverage probability expressions and potential throughput expressions at the terminals in this system.

Based on the analysis model and simulation results, we propose a scheduling scheme to exploit D2D interference to achieve throughput fairness.

The rest of this article is organized as follows. Section 2 describes the proposed system model to formulate the optimization problem. In Section 3, we use an OrthoMADS algorithm to solve the MINLP optimization problem. Section 4 provides a detailed analysis of this paper in terms of the experimental study. Finally, Section 5 gives conclusions and points out future research directions.

\section{NETWORK MODEL AND PROBLEM FORMULATION 2.1 Network Model}

We focus on a network using only the unlicensed spectrum. In this system, LTE users can switch to D2D mode for communication with the assistance of evolutional NodeB (eNB). Other transmit sets include a single eNB and multiple $\mathrm{Wi}-\mathrm{Fi}$ access points (APs). The eNB is located at the center with a disk coverage area. Wi-Fi APs and D2D transmitters are distributed on this plane according to Poisson distribution with intensities $\lambda_{d}, \lambda_{w}$ Each type of UE is already attached to its AP and D2D transmitter, and their position is subject to the uniform distribution. Unlike the above literature, we focus on the situation where cochannel interference has already occurred. We only consider a downlink scenario. D2D model selection is a difficult problem in D2D networks. In this paper, we use a distance-based model selection scheme such as that in [25], in which every successful D2D pair has a maximum distance of $r_{d}$ between nodes, and the possibility of pairing more than two D2D users with the reference user ignored in this work. Fig. 1 illustrates the network model of this proposed work involving all entities discussed above.

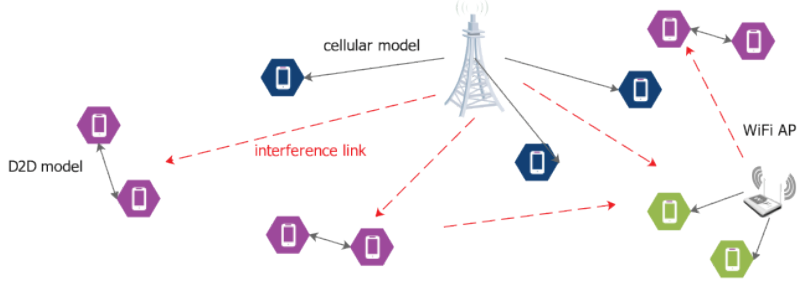

Figure 1 System Model

Under this assumption, a communication participant can be generally represented as a marked Poisson point process (PPP), and the different types of PPP sets are independent. The general PPP is denoted as:

$\Phi=\left\{X_{i}, r_{i}, P_{i}\right\}$

where:

(1) $\left\{X_{i}\right\}$ indicates the locations of the unlicensed band transmitters such as D2D transmitters and Wi-Fi APs;

(2) $\left\{r_{i}\right\}$ indicates the link distance of various types of communication in this scenario;
(3) $\left\{P_{i}\right\}$ indicates the transmit power of $X_{i}$.

In this paper, we only consider the downlink. The downlink channel model includes a power-law propagation model and Rayleigh fading. Under this assumption, the typical receiver received power is:

$p_{r}=p_{t} \cdot h \cdot r^{-\alpha}$

where $p_{t}$ is the transmit power; $h$ is the fading coefficient, which is exponential with $1 / \mu$ and is denoted as $h \sim \exp (\mu)$; $r$ is the link distance between receiver and transmitter, and $\alpha$ is the path-loss exponent.

The transmission powers are assumed to be $P_{c}$ at eNB, $P_{d}$ at D2D transmitters and $P_{w}$ at Wi-Fi APs.

Table 1 Notation and symbols used in the paper

\begin{tabular}{|c|l|}
\hline Symbol & Definition/Explanation \\
\hline$\Phi$ & the general set of transmitters \\
\hline$\Phi_{d}$ & the active set of D2D transmitters with $\lambda_{d}$ \\
\hline$\Phi_{w}$ & the active set of Wi-Fi APs with $\lambda_{w}$ \\
\hline$x_{i}^{*}$ & transmit node; $*$ indicates D2D and AP \\
\hline$y_{i}^{*}$ & receive node; $*$ indicates D2D and AP \\
\hline$r_{c}$ & $\begin{array}{l}\text { link distance between cellular user equipment (CUE) and } \\
\text { eNB }\end{array}$ \\
\hline$r_{d}$ & $\begin{array}{l}\text { link distance between D2D user equipment (DUE) in a } \\
\text { single D2D pair }\end{array}$ \\
\hline$r_{w}$ & $\begin{array}{l}\text { link distance between Wi-Fi user equipment (WUE) and a } \\
\text { Wi-Fi access point }\end{array}$ \\
\hline$r_{\text {inte }}^{*}$ & $\begin{array}{l}\text { distance between a typical receiver and an interferer; }{ }^{*} \text { is } \\
\text { the type of the receiver }\end{array}$ \\
\hline$P_{c}$ & eNB transmit power \\
\hline$P_{d}$ & D2D transmit power \\
\hline$P_{w}$ & Wi-Fi access point transmit power \\
\hline$N_{0}$ & AWGN noise $\sigma^{2}$ \\
\hline$h$ & $\begin{array}{l}\text { channel fading parameter, whereh } \\
\text { into is the interfering } \\
\text { channel fading parameter; h } \sim \text { exp }(\mu)\end{array}$ \\
\hline$\alpha$ & path-loss exponent \\
\hline$\theta_{c}$ & threshold SINR at CUE \\
\hline$\theta_{d}$ & threshold SINR at DUE \\
\hline$\theta_{w}$ & threshold SINR at Wi-Fi Station $(\mathrm{STA})$ \\
\hline$\mu$ & Rayleigh fading coefficient of a link with $1 / \mu$ \\
\hline & \\
\hline
\end{tabular}

\subsection{Coverage Probabilityand Performance Metrics}

For the purposes of mathematical tractability, we consider all users around their associated transmitter. Link distance obeys uniform distribution. The probability density function of distance is

$f(r)=\frac{2 r}{R^{2}}$

where $R$ is the coverage radius of each communication. Subscripts will be used to specify this in future discussions, such as the maximum link distance in Wi-Fi is $R_{w}$, and the D2D maximum link distance is $R_{d}$.

First, we analyze the average coverage expression of cellular users after the introduction of D2D. On the condition that fading is independent and identically distributed (i.i.d) and that the distance between eNB and a typical cellular user is $r_{c}$, the coverage probability for CUE is: 


$$
\begin{aligned}
& p_{c o v}^{c}=\mathbb{E}\left[\mathbb{P}\left[\mathrm{SINR}_{c}>\theta_{c} \mid r_{c}\right]\right]= \\
& =\int_{r_{c}>0} \mathbb{P}\left[\frac{P_{c} h r_{c}^{-\alpha}}{N_{0}+I_{r}}>\theta_{c} \mid r_{c}\right] \frac{2 r_{c}}{R^{2}} \mathrm{~d} r_{c}
\end{aligned}
$$

For cellular users, interference is generated by D2D transmitters and Wi-Fi APs. When the channels are allocated, the SINR of the $i$-th CUE is:

$$
\operatorname{SINR}_{i}^{c}=\frac{P_{c} h r_{c}^{-\alpha}}{\sum_{S T A_{i} \in \Phi_{W}} P_{w} h_{i}^{w} r_{\text {inte }}^{w-\alpha}+\sum_{d u e_{i} \in \Phi_{D}} P_{d} h_{i}^{d} r_{\text {inte }}^{d-\alpha}+N_{0}}
$$

Then, the total interference is calculated by Eq. (5):

$$
I_{\text {sum }}^{c}=\sum_{\Phi_{d i}} P_{d} \cdot h_{i}^{w} \cdot r_{\text {inte }}^{d-\alpha}+\sum_{\Phi_{w i}} P_{w} \cdot h_{i}^{w} \cdot r_{\text {inte }}^{w-\alpha}
$$

We denote $r_{\text {inte }}^{d}$ and $r_{\text {inte }}^{w}$ as the distance from an interfering D2D transmitter and interfering APs, respectively, to the typical CUE. We assume that $h \sim \exp (\mu)$; then:

$$
\begin{aligned}
& \mathbb{P}\left[h>P_{c}^{-1} \theta_{c} r_{c}^{\alpha}\left(N_{0}+I_{\text {sum }}\right) \mid r_{c}\right]= \\
& =\int_{P_{c}{ }^{-1} \theta_{c} r_{c}{ }^{\alpha}\left(N_{0}+I_{\text {sum }}\right)} \mathrm{e}^{-\mu x} \mathrm{~d} \mu x=
\end{aligned}
$$

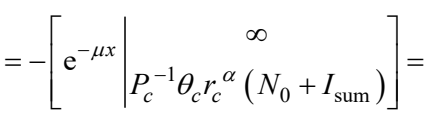

$$
\begin{aligned}
& =-\left[\mathrm{e}^{-\infty}-\mathrm{e}^{-\mu P_{c}^{-1} \theta_{c} r_{c}^{\alpha}\left(N_{0}+I_{\text {sum }}\right)}\right]= \\
& =\mathrm{e}^{-\mu P_{c}^{-1} \theta_{c} r_{c}^{\alpha}\left(N_{0}+I_{\text {sum }}\right)}= \\
& =\mathrm{e}^{-\mu P_{c}^{-1} \theta_{c} r_{c}^{\alpha} N_{0}} \cdot \mathrm{e}^{-\mu P_{c}^{-1} \theta_{c} r_{c}^{\alpha} I_{\text {sum }}} \\
& =\exp \left(-\mu P_{c}^{-1} \theta_{c} r_{c}^{\alpha} N_{0}\right) \mathcal{L}_{I_{D}}\left(\mu P_{c}^{-1} \theta_{c} r_{c}^{\alpha}\right) \mathcal{L}_{I_{W}}\left(\mu P_{c}^{-1} \theta_{c} r_{c}^{\alpha}\right)
\end{aligned}
$$

Let $s=\mu P_{c}^{-1} \theta_{c} r_{c}^{\alpha}$. Using the Laplace transform, we have:

$$
\begin{aligned}
\mathcal{L}_{I_{D}}(s) & =\mathbb{E}_{\Phi_{d}}\left[\prod_{i \in \Phi_{D}} \mathbb{E}_{h_{d}}\left(\exp \left(-s \cdot P_{d} \cdot h_{i}^{d} \cdot r_{\text {inte }}^{d i}-\alpha\right)\right)\right] \\
& =\mathbb{E}_{\Phi_{d}}\left[\prod_{i \in \Phi_{d}} \frac{\mu}{s P_{d} r_{\text {inte }}^{d i-\alpha}+\mu}\right] \\
& =\exp \left[-\lambda_{d} \int_{\mathbb{R}^{2}}\left(1-\frac{\mu}{s P_{d} r_{\text {inte }}^{d i-\alpha}+\mu}\right) d r_{\text {inte }}^{d i}\right] \\
& =\exp \left[-\pi \lambda_{d} m_{d}^{\frac{2}{\alpha}} \cdot \frac{\frac{2 \pi}{\alpha}}{\sin \left(\frac{2 \pi}{\alpha}\right)}\right]
\end{aligned}
$$

where $m_{d}=\mu P_{d} P_{c}^{-1} \theta_{c} r_{c}^{-\alpha}$. Similarly, the Laplace transform of the interference from APs can be calculated by Eq. (8):
$\mathcal{L}_{I_{W}}(s)=\exp \left[-\pi \lambda_{w} m_{w}^{\frac{2}{\alpha}} \cdot \frac{\frac{2 \pi}{\alpha}}{\sin \left(\frac{2 \pi}{\alpha}\right)}\right]$ Eq. (9):

The coverage probability of CUE can be expressed as

$$
\begin{aligned}
& p_{c o v}^{c}\left(\theta_{c}, \lambda_{d}, \lambda_{w}\right)=\int_{r>0} \exp \left(-\mu P_{c}^{-1} \theta_{c} r_{c}^{\alpha} N_{0}\right) . \\
& \cdot \exp \left[-\pi \lambda_{d} m_{d}^{\frac{2}{\alpha}} \cdot \frac{2 \pi}{\alpha \sin \left(\frac{2 \pi}{\alpha}\right)}\right] \\
& \cdot \exp \left[-\pi \lambda_{w} m_{w}^{\frac{2}{\alpha}} \cdot \frac{2 \pi}{\alpha \sin \left(\frac{2 \pi}{\alpha}\right)}\right] \cdot \frac{2 r_{c}}{R^{2}} d r_{c}
\end{aligned}
$$

when $N_{0} \rightarrow 0$, which means that in an interferencelimited scenario, the coverage probability can be calculated by Eq. (10):

$$
p_{\mathrm{cov}}^{c}\left(\theta_{c}, \lambda_{d}, \lambda_{w}\right)=\frac{\exp \left(-\pi R^{2}\left(\mu P_{c}^{-1} \theta_{c}\right)^{2 / \alpha} \frac{2 \pi}{\alpha \sin (2 \pi / \alpha)} \cdot\left(\lambda_{d} P_{d}^{2 / \alpha}+\lambda_{w} P_{w}^{2 / \alpha}\right)\right)-1}{-\pi R^{2}\left(\mu P_{c}^{-1} \theta_{c}\right)^{2 / \alpha} \frac{2 \pi}{\alpha \sin (2 \pi / \alpha)} \cdot\left(\lambda_{d} P_{d}^{2 / \alpha}+\lambda_{w} P_{w}^{2 / \alpha}\right)}
$$

Note that when analyzing the coverage probability of a typical D2D receiver and a typical STA, their distances to the eNB are similar to the distance between CUE and the eNB. The D2D pairs have a maximum distance $R_{d}$, and we assume that the D2D link distance distribution is uniform. Similarly, the received SINR of Wi-Fi users is:

$$
\begin{aligned}
& \operatorname{SINR}_{i}^{w}= \\
& =\frac{P_{w} h_{w} r_{w}{ }^{-\alpha}}{\sum_{S T A_{j} \in \Phi_{w} \backslash\left\{x_{i}\right\}} P_{w} h_{i}^{w} r_{\text {inte }}^{w-\alpha}+P_{L} h_{i}^{L} r_{\text {inte }}^{L-\alpha}+\sum_{d u e_{i} \in \Phi_{d}} P_{d} h_{i}^{d} r_{\text {inte }}^{d-\alpha}+N_{0}}
\end{aligned}
$$

and the SINR of DUE is:

$$
\begin{aligned}
& \operatorname{SINR}_{i}^{d}= \\
& =\frac{P_{d} h_{d} r_{d}^{-\alpha}}{\sum_{S T A_{i} \in \Phi_{w}} P_{w} h_{i}^{w} r_{\text {inte }}^{w-\alpha}+P_{c} h_{i}^{L} r_{\text {inte }}^{L-\alpha}+\sum_{d u e_{j} \in \Phi_{d} \backslash\left\{x_{i}\right\}} P_{d} P h_{i}^{d} r_{\text {inte }}^{d-\alpha}+N_{0}}
\end{aligned}
$$

Thus, the approximate expressions for the D2D user coverage probability and STA coverage probability are:

$$
\begin{gathered}
p_{c o v}^{d}\left(\theta_{d}, \lambda_{d}, \lambda_{w}\right)=\frac{\exp \left(\left(-\pi R_{d}^{2} \frac{2 \pi}{\alpha \sin (2 \pi / \alpha)}\right)\right.}{-\pi R_{d}^{2} \frac{2 \pi}{\alpha \sin (2 \pi / \alpha)}} . \\
\frac{\left.\left(\left[\left(\mu \theta_{d}\right)^{2 / \alpha} \cdot\left(\lambda_{d}+\lambda_{w}\left(P_{w} P_{d}^{-1}\right)^{2 / \alpha}+\lambda_{c}\left(P_{c} P_{d}^{-1}\right)^{2 / \alpha}\right)\right]\right)\right)-1}{\left[\left(\mu \theta_{d}\right)^{2 / \alpha} \cdot\left(\lambda_{d}+\lambda_{w}\left(P_{w} P_{d}^{-1}\right)^{2 / \alpha}+\lambda_{c}\left(P_{c} P_{d}^{-1}\right)^{2 / \alpha}\right)\right]}
\end{gathered}
$$




$$
\begin{aligned}
& p_{c o v}^{w}\left(\theta_{w}, \lambda_{w}, \lambda_{d}\right)= \\
& \frac{\exp \left(\left(-\pi R_{w}{ }^{2} \frac{2 \pi}{\alpha \sin (2 \pi / \alpha)}\right)\right.}{-\pi R_{w}{ }^{2} \frac{2 \pi}{\alpha \sin (2 \pi / \alpha)}} \cdot \\
& \frac{\left.\left(\left[\left(\mu \theta_{w}\right)^{2 / \alpha} \cdot\left(\lambda_{w}+\lambda_{d}\left(P_{d} P_{w}{ }^{-1}\right)^{2 / \alpha}+\lambda_{c}\left(P_{c} P_{w}{ }^{-1}\right)^{2 / \alpha}\right)\right]\right)\right)-1}{\left[\left(\mu \theta_{w}\right)^{2 / \alpha} \cdot\left(\lambda_{w}+\lambda_{d}\left(P_{d} P_{w}{ }^{-1}\right)^{2 / \alpha}+\lambda_{c}\left(P_{c} P_{w}^{-1}\right)^{2 / \alpha}\right)\right]}
\end{aligned}
$$

The Eq. (10) and Eq. (14) show that both the Wi-Fi users' coverage probability and cellular users' coverage probability are inversely correlated with the density of active D2D pairs and transmit power. In the performance evaluation, we selected the potential throughput as the evaluation criterion. The potential throughput is defined as in [26], as:

$$
\tau(P, \lambda, \theta)=\lambda \log _{2}(1+\theta) P_{\mathrm{cov}}
$$

When the $\theta$ is fixed, this metric can be used to study the ratio of transmitter density and transmit power to throughput. The unit of this metric is $\mathrm{bps} / \mathrm{Hz} / \mathrm{m}^{2}$, and by combining the area and bandwidth of the system, the throughput can be calculated through this metric. According to the (13) and (14), when $P_{c}, P_{w}$ and $\lambda_{w}$ are constant, the Wi-Fi throughput is affected by $\lambda_{d}$ and $P_{d}$. The expression shows that we can adjust the throughput difference between the two different systems by adjusting the number of D2D and its transmit power.

\subsection{Problem Formulation}

Based on the analytical results, the potential throughput of LTE users and Wi-Fi users will be affected by the intensity of active D2D pairs $\left(\lambda_{d}\right)$ and its transmit power $P_{d}$. According to 3GPP's requirements for fairness, the main point of our proposed scheduling scheme is that when D2D is introduced, the fairness of existing systems will be better than before. To guarantee perfect links in Wi$\mathrm{Fi}$, we should control the number of unlicensed channels allocated to D2D pairs $\lambda_{d}$ and the D2D transmit power $P_{d}$.

The performance of the cellular user can be enhanced by introducing D2D communication, that is, by increasing $\lambda_{d}$. Furthermore, for D2D users, increasing transmit power means obtaining a better experience. However, as $\lambda_{d}$ and $P_{d}$ increase, the achievable coverage probability of WiFi will decrease. Therefore, the scheduling parameters $\lambda_{d}$ and $P_{d}$ should be carefully designed when considering the impacton Wi-Fi. We consider D2D users and LTE-U as generalized cellular users. Our objective is to minimize the potential throughput difference between cellular and Wi-Fi systems. The scheduling problems about $\lambda_{d}$ and $P_{d}$ can be expressed as the following optimization problems:

$$
\begin{gathered}
\min _{\lambda_{d}, P_{d}}\left[\tau_{c}\left(P_{d}, \lambda_{c}^{d}, \theta_{c}\right)+\tau_{d}\left(P_{d}, \lambda_{d}, \theta_{d}\right)-\tau_{w}\left(P_{d}, \lambda_{w}^{d}, \theta_{w}\right)\right]^{2} \\
\text { s.t: } \quad \begin{array}{l}
\text { C1: } \lambda_{\max } \geq \lambda_{d} \geq 1 /|B| \\
\text { C2: } p_{\max } \geq p_{d} \geq p_{\min } \\
\text { C3: } \lambda_{d}=m \cdot \lambda_{e N B} \quad m \in \mathbb{Z}
\end{array}
\end{gathered}
$$

Eq. (16) is the objective function and Eq. (17) is the constraint. Constraint $\mathrm{C} 1$ implies that in this scenario, there exists at least one D2D pair, and the D2D density is maximal. Constraint $\mathrm{C} 2$ implies that the D2D transmit power should be within the specified interval. Constraint C3 means the current D2D transmitter density is an integer multiple of eNB density. Considering that the transmit power of the $\mathrm{D} 2 \mathrm{D}$ is a continuous variable, the aforesaid scheduling problem is an MINLP problem.

\section{ORTHO-MADS BASED SCHEDULING SCHEME}

In the above optimization problem, the derivative of the objective function is complicated, and the derivative information is not available. A derivative-free optimization algorithm can solve this type of problem.

Mesh adaptive direct search is an iterative pattern search algorithm. The iterative process includes two steps: a search step and a detection step. The search step selects a point sequence to identify a feasible region containing a local optimum; that is, the search step is a process of global search in the entire variable space. The algorithm selects a limited number of test points on the grid, compares their objective function values, and finds the test point with the smallest function value. The detection step is a local search in the neighborhood of the test point according to the detection direction to accurately find the best advantage. The direction matrix generation proceeds as follows:

The trial points are generated by Eq. (18):

$M_{k}=\left\{x+\Delta_{k}^{m} D y \quad: \mathrm{y} \in \mathbb{N}^{n}\right\}$

and the poll stage trial points are created by Eq. (19):

$P_{k}=\left\{x_{k}+\Delta_{k}^{m} \mathrm{~d}: \mathrm{d} \in D_{k}\right\}$

where $D$ is the set of directions, and $\mathrm{n}$ is the number of directions. The direction set $D$ is generated according to the method in reference [27]. The parameter $\lambda_{d}=M \cdot \lambda_{c}$ where $\lambda_{c}=1 /$ area which represents the average number of eNB. This value is much smaller than the value of the transmit power. Since the MADS algorithm is sensitive to the initial poll size and mesh size, it is different from reference [27], for $\lambda_{d}$ and $P_{d}$, the initial poll size parameter and mesh size parameter is generated by Eq. (20):

$\Delta_{0}^{m}=\Delta_{0}^{p}=\frac{U-L}{10}$

where $U$ is the upper bound, and $L$ is the lower bound for each variable.

\section{SIMULATION RESULTS}

This section comprises the simulation setup and comparative analysis. The critical specifications involved in the execution of our proposed simulation work are discussed in the simulation setup section. To validate the achievement of this proposed work, we compared the results obtained against previous algorithms in the comparative analysis section. 
Table 2 Ortho-MADS-based scheduling scheme

\begin{tabular}{|c|l|}
\hline \multicolumn{2}{|c|}{ Ortho-MADS-based Scheduling Scheme } \\
\hline \multicolumn{2}{|c|}{ Input: Initial points $x_{0}, \theta_{d}, \theta_{c}, \theta_{w}, P_{c}, P_{w}, \lambda_{w}$} \\
\hline Output: $\lambda_{d}, P_{d}$ \\
\hline 2: & Initialization \\
\hline 3: & Shoose $x_{0} \in \mathbb{R}, \Delta_{0}^{m}=\Delta_{0}^{p}=1 l_{0}$ \\
\hline 4: & Parameter Update \\
\hline 5: & Set the mesh size $\Delta_{k}^{m} \leftarrow \min \left\{1,4^{-l_{k}}\right\}$ and $\Delta_{k}^{p} \leftarrow 2^{-l_{k}} l_{k} \in \mathbb{Z}$ \\
\hline 6: & Search \\
\hline 7: & Select a finite subset $S^{k}$ of $M^{k}$ \\
\hline 8: & Obtain the value of $f\left(x_{k}\right)$ and $f\left(x_{k}+s_{k}\right)$ \\
\hline 9: & if $f\left(x_{k}+s_{k}\right)<f\left(x_{k}\right)$ for some $x_{k}+s_{k} \in S^{k}$ \\
\hline 10: & Set $x_{k+1} \leftarrow x_{k}+s_{k}$ and $l_{k+1} \leftarrow l_{k}-1$ and go to [Termination] \\
\hline 11: & Go to [Poll] \\
\hline 12: & Poll \\
\hline 13: & Select a positive spanning set $D_{k}^{p}$ such that $x_{k}+\Delta_{k}^{m} d \in F^{k} \quad$ for \\
& all $d \in D_{k}^{p}$ \\
\hline 14: & Obtain the value of $f\left(x_{k}\right)$ and $f\left(x_{k}+s_{k}\right)$ \\
\hline 15: & Success \\
\hline 16: & if $f\left(x_{k}+s_{k}\right)<f\left(x_{k}\right)$ for some $s_{k}=\Delta_{k}^{m} d_{k} \in\left\{\Delta_{k}^{m} d: d \in D\right\}$ \\
\hline 17: & Set $x_{k+1} \leftarrow x_{k}$ and $l_{k+1} \leftarrow l_{k}-1$ \\
\hline 18: & Failure \\
\hline 19: & if $f\left(x_{k}+s_{k}\right) \geq f\left(x_{k}\right)$ \\
\hline 20: & Set $x_{k+1} \leftarrow x_{k}$ and $l_{k+1} \leftarrow l_{k}+1$ \\
\hline 21: & Termination \\
\hline 22: & Set $k \leftarrow k+1$ and go to Parameter Update \\
\hline 23: & otherwise, stop \\
\hline
\end{tabular}

\subsection{Simulation Setup}

First, a Monte Carlo simulation is established to verify the accuracy of the calculated expressions in Section 2. In the simulation, the density of APs is $10 \lambda_{\mathrm{c}}$, and the density of D2Ds is $20 \lambda_{\mathrm{c}}$. The CUE is generated randomly with a uniform distribution. The D2D and Wi-Fi transmitters are generated randomly with a Poisson distribution over the plane, and the receivers are created with uniform distribution with their respective distance limits. Tab. 3 lists the main parameter settings in the simulation experiment. However, the specifications used for our simulation are not limited to these; this work also involves many modules that support the real output of the performance. More simulation parameters will be noted in the specific analysis.

Table 3 Main simulation parameters

\begin{tabular}{|c|c|}
\hline Parameter & Value \\
\hline eNB TX power & $46 \mathrm{dBm}$ \\
\hline Wi-Fi AP TX power & $23 \mathrm{dBm}$ \\
\hline Max. D2D TX power & $23 \mathrm{dBm}$ \\
\hline Min. D2D TX power & $\mathrm{dBm}$ \\
\hline Cell radius & $1000 \mathrm{~m}$ \\
\hline Max. D2D link distance & $20 \mathrm{~m}$ \\
\hline Max. Wi-Fi link distance & $100 \mathrm{~m}$ \\
\hline Noise power & $-96 \mathrm{dBm}$ \\
\hline System bandwidth & $20 \mathrm{MHz}$ \\
\hline Path-loss exponent & 4 \\
\hline Number of Monte Carlo simulation runs & 1000000 \\
\hline
\end{tabular}

\subsection{Validation of Analytical Results}

In this part, we validate the accuracy and assumptions of our analytical model by simulation experiments.
In the analysis, we approximate the SINR distribution by a SIR distribution. Fig. 2 plots the simulation results of the AWGN scenario with the results of the Monte Carlo analysis model. The simulation results with and without noise are similar, indicating that Eq. (10), Eq. (13) and Eq. (14) can be used for subsequent analysis. Fig. 3 plots the noise-free scene. As seen from the figures, the calculation results of the coverage probability analytical model in Eq. (10), Eq. (13) and Eq. (14) are basically consistent with the corresponding simulation results.

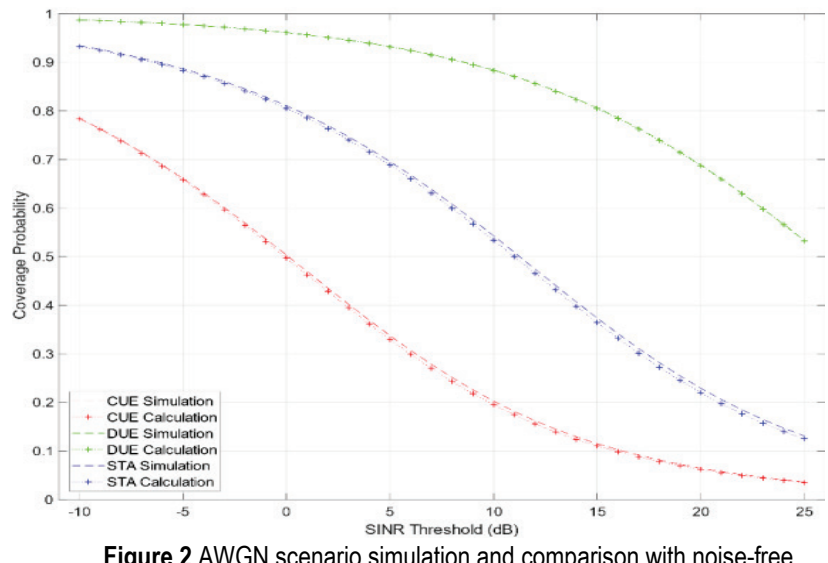

Figure 2 AWGN scenario simulation and comparison with noise-free calculation results

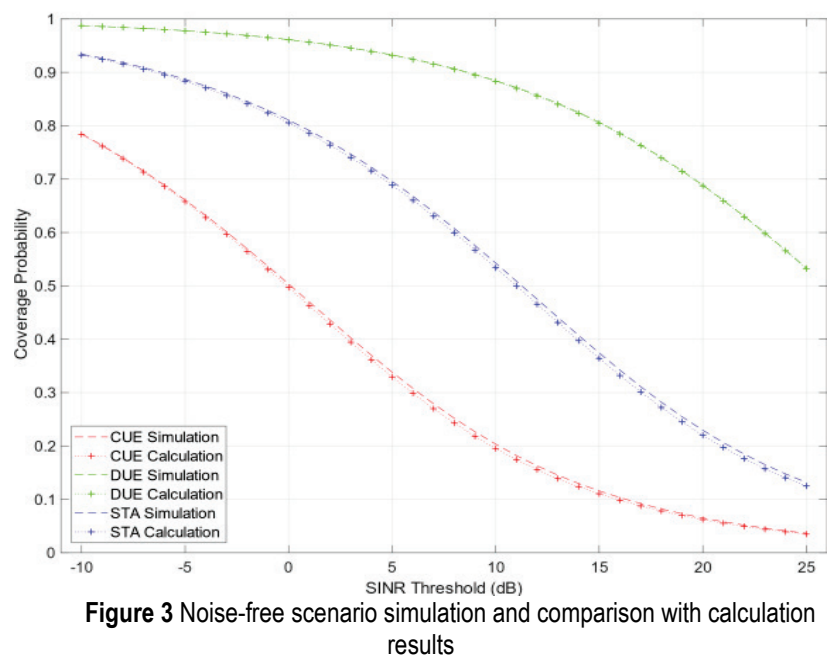

\subsection{Fairness Assessment of the Proposed Scheme}

In this section, we provide numerical results of simulation experiments to evaluate the fairness performance of the proposed scheme. To better assess the performance of the proposed schemes, we subdivided the simulation into three cases: (1) D2D-U users have the lowest priority, which means $\theta_{d}<\min \left(\theta_{c}, \theta_{w}\right)$; (2) D2D-U, LTE-U, and Wi-Fi users have the same priority, which means $\theta_{d}=\theta_{c}=\theta_{w}$; (3) D2D-U users have the highest priority, which means $\theta_{d}>\max \left(\theta_{c}, \theta_{w}\right)$; in cases 1 and $3, \theta_{c}$ $=15 \mathrm{~dB}, \theta_{w}=20 \mathrm{~dB}$. As a comparison, we chose the commonly used penalty function (PF) method to compare with these schemes.

Fig. 4 shows appropriate $\left(\lambda_{d}, P_{d}\right)$ can make the difference in throughput between systems small enough. In Fig. 4, the relationship between throughput difference and $\left(\lambda_{d}, P_{d}\right)$ is not monotonic when $\lambda_{d}$ and $P_{d}$ increase to a 
particular set of values, and the throughput difference can be made sufficiently small.

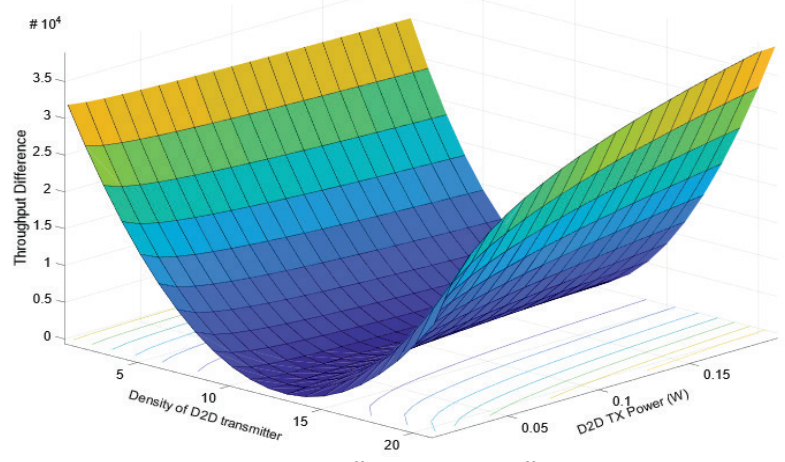

Figure 4 Throughput difference under different $\lambda_{d}$ and $P_{d}$

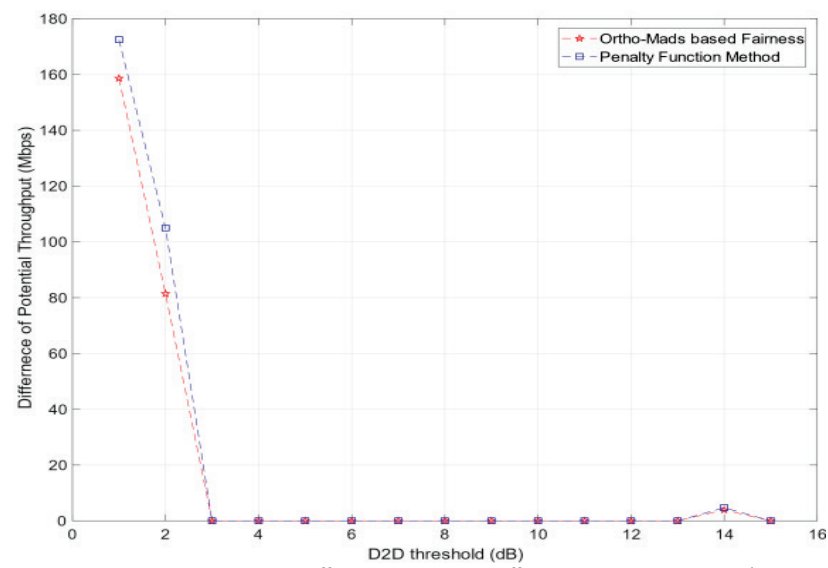

Figure 5 Throughput difference under a different scheme in case 1

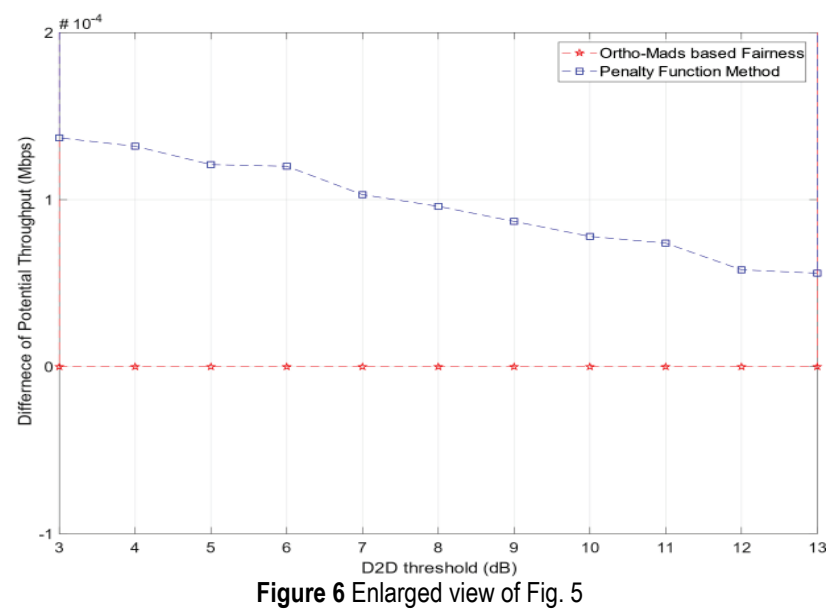

Fig. 5 illustrates the scheduling scheme performance for different D2D thresholds in case 1, and Fig. 6 is an enlarged part of Fig. 5. It should be noted that although the result is shown as zero, the calculation result is not truly zero; when the throughput difference is small enough, we will approximate it as zero. For example, in the proposed scheme when $\lambda_{d}=12$ and $P_{d}=0,01186$, the difference value is $1,48911 \mathrm{e}-10 \mathrm{Mbps}$. When $\lambda_{d}<3$, the performance of both schemes is significantly larger than $\theta_{d}$. This is because when the threshold of D2D is small, all the D2D transmitter's data rates are low, and even if all D2D users are allocated an unlicensed spectrum, they will not have sufficient impact on the throughput difference. When $3 \mathrm{~dB} \leq \theta_{d} \leq 13 \mathrm{~dB}$, in the PF method difference in two systems, is in the range of $0,000161 \mathrm{Mbps}$ to 0,0000486 Mbps. In our method, the difference is 0 . When $13<\theta_{d} \leq 15$, both methods cannot achieve zero difference, but our method is closer to 0 than the PF method. The results show that the proposed scheme can achieve better fairness at the same D2D rate.

Fig. 7 shows the scheduling scheme performance at different D2D thresholds in case 2, in which all users have the same priority. We can see that for both schemes, as $\theta_{d}$ increases, the throughput difference reduces. In addition, when $1 \mathrm{~dB} \leq \theta_{d} \leq 5 \mathrm{~dB}$, the same phenomenon occurs in case 2. The PF method keeps a better balance than our method. But the gap between the two methods is less than $0,00186 \mathrm{Mbps}$, which is acceptable. However, in case 2, when $\theta_{d} \leq 3 \mathrm{~dB}$, the difference is significantly smaller than it is in case 1 . This is because in case 2, both types of users have the same priority, which means $\theta_{d}=\theta_{c}=\theta_{w}$, and eNB and Wi-Fi APs have the same data rate, which is lower than that in case 1 . Furthermore, when $\theta_{d}>3 \mathrm{~dB}$, the figure shows that our method is closer to 0 than the PF method, which means our method achieves a better balance than the PF method.

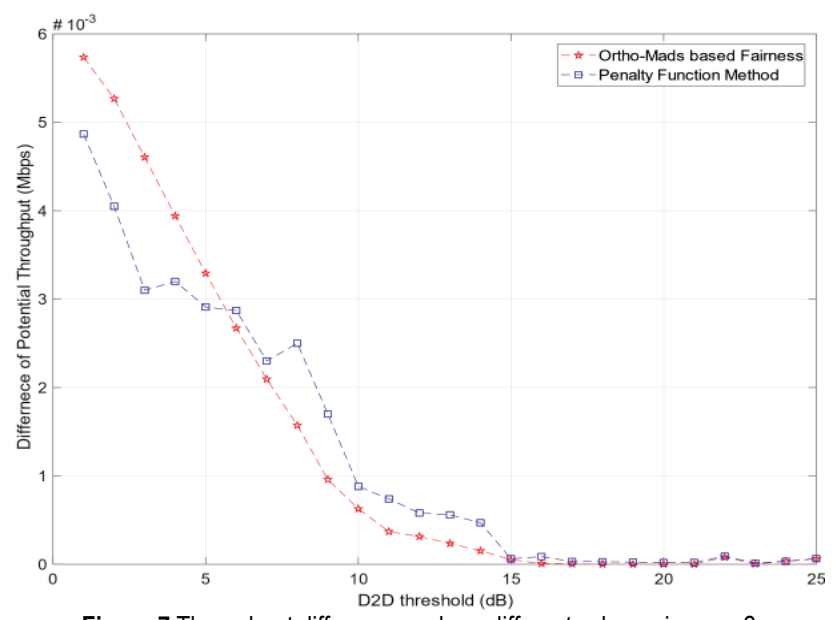

Figure 7 Throughput difference under a different scheme in case 2

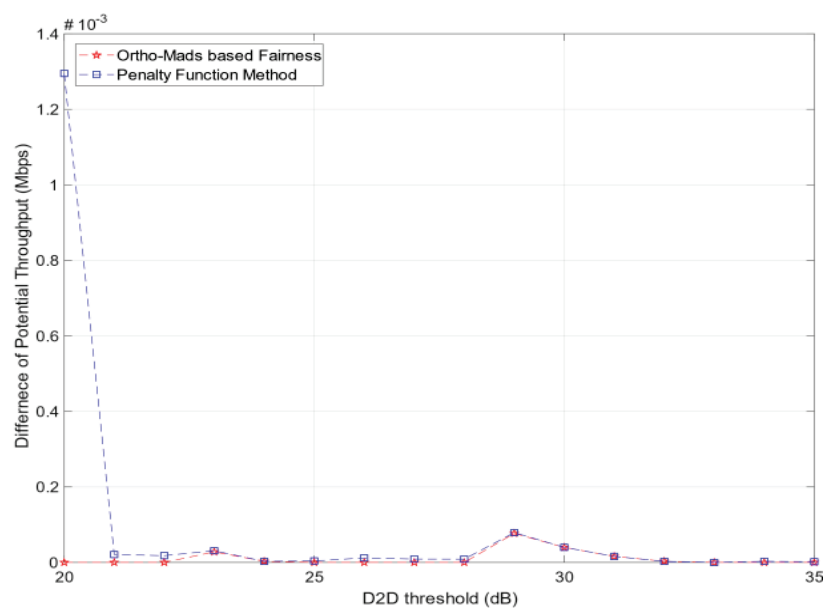

Figure 8 Throughput difference under a different scheme in case 3

Fig. 8 shows the simulation results in case 3 .We can observe that when D2D has a high transmission rate, both schemes can maintain a small difference in throughput. However, compared with the PF method, the proposed scheme performs better or the same as the PF method in 
case 3 . When $\theta_{d}=20 \mathrm{~dB}$, we can see that in our method, the throughput of cellular is equal to the Wi-Fi system, but in the PF method, the difference in throughput is 0,001295 Mbps. When $\theta_{d}$ changing from $21 \mathrm{~dB}$ to $35 \mathrm{~dB}$, although our method is not far from the PF method, it performs better than the PF method in the above range. In summary, in scenarios where there is a rate requirement, as the threshold value changes, the range of throughput difference of the proposed scheme is smaller than that of the PF method, which indicates that the proposed scheme has better adaptability than previous work.

As the above experimental results show, the proposed scheme performs better or the same as the PF method in all three scenarios.

\section{CONCLUSIONS}

In this paper, a fairness enhancement schedule scheme under the coexistence of LTE and Wi-Fi over an unlicensed band by exploiting D2D interference is proposed. This process aims to improve the fairness of the unlicensed band coexistence scenario. We proposed a framework for analyzing such situations through stochastic geometry, and we derive a closed-form expression for the average coverage probability and potential throughput. In fairness based schedule problem, the difference in potential throughput is used to evaluate the performance of the framework. The model shows that the throughput fairness of the system can be achieved by adjusting the D2D density and transmit power. Based on this, we turn the fairnessenhanced scheduling scheme into an MINLP problem and use the Ortho-MADS algorithm to solve the problem. In the simulation, we verified the proposed system in three different cases. The numerical result shows when D2D mode is introduced, interference from D2D can instead reduce the difference in throughput between the cellular and the Wi-Fi system. In unlicensed band coexistence networks, this study provides a new perspective on interference.

The main limitation of the current scheme is that the transmitting data rate of all users is assumed to be a fixed value set in advance. An important direction in future research is to add link adaptation technology to the analysis model.

\section{Acknowledgements}

This work was supported in part by the Natural Science Foundation of China under Grant 611772088, Grant 11801042 and Grant 11771059, and in part by the Changsha University of Science and Technology under Grant K1705081.

\section{REFERENCES}

[1] Wang, X., Mao, S., \& Gong, M. X. (2017). A survey of LTE Wi-Fi coexistence in unlicensed bands. GetMobile: Mobile Computing and Communications, 20(3), 17-23. https://doi.org/10.1145/3036699.3036705

[2] Cano, C., Lopez-Perez, D., Claussen, H., \& Leith, D. J. (2016). Using LTE in unlicensed bands: Potential benefits and coexistence issues. IEEE communications magazine, 54(12), 116-123.
https://doi.org/10.1109/MCOM.2016.1500413CM

[3] Shang, B., Zhao, L., \& Chen, K. C. (2017, May). Enabling device-to-device communications in LTE-unlicensed spectrum. 2017 IEEE International Conference on Communications (ICC), 1-6. https://doi.org/10.1109/ICC.2017.7997134

[4] Asadi, A., Wang, Q., \& Mancuso, V. (2014). A survey on device-to-device communication in cellular networks. IEEE Communications Surveys \& Tutorials, 16(4), 1801-1819. https://doi.org/10.1109/COMST.2014.2319555

[5] AlQahtani, S. A. (2018). Modeling and performance analysis of unlicensed bands MAC strategy in multi-channel LTE-A networks with M2M/H2H coexistence. Wireless Networks, 24(6), 1965-1978. https://doi.org/10.1007/s11276-017-1449-6

[6] Ma, Y. \& Kuester, D. G. (2017, January). MAC-layer coexistence analysis of LTE and WLAN systems via listenbefore-talk. 2017 14th IEEE Annual Consumer Communications \& Networking Conference (CCNC), 534541. https://doi.org/10.1109/CCNC.2017.7983164

[7] Zhang, Q., Wang, Q., Feng, Z., \& Yang, T. (2016). Design and performance analysis of a fairness-based license-assisted access and resource scheduling scheme. IEEE Journal on Selected Areas in Communications, 34(11), 2968-2980. https://doi.org/10.1109/JSAC.2016.2614950

[8] Ko, H., Lee, J., \& Pack, S. (2016). A fair listen-before-talk algorithm for coexistence of LTE-U and WLAN. IEEE Transactions on Vehicular Technology, 65(12), 1011610120. https://doi.org/10.1109/TVT.2016.2533627

[9] Chen, Q., Yu, G., \& Ding, Z. (2016). Optimizing unlicensed spectrum sharing for LTE-U and WiFi network coexistence. IEEE Journal on Selected Areas in Communications, 34(10), 2562-2574. https://doi.org/10.1109/JSAC.2016.2604998

[10] Chatterjee, S., Abdel-Rahman, M. J., \& MacKenzie, A. B. (2017). Optimal distributed allocation of almost blank subframes for LTE/WiFi coexistence. 2017 15th International Symposium on Modeling and Optimization in Mobile, Ad Hoc, and Wireless Networks (WiOpt), 1-6. https://doi.org/10.23919/WIOPT.2017.7959866

[11] Kwon, H. J., Jeon, J., Bhorkar, A., Ye, Q., Harada, H., Jiang, Y., \& Oh, J. (2016). Licensed-assisted access to unlicensed spectrum in LTE release 13. IEEE communications magazine, 55(2), 201-207. https://doi.org/10.1109/MCOM.2016.1500698CM

[12] Mehrnoush, M., Roy, S., Sathya, V., \& Ghosh, M. (2018). On the fairness of Wi-Fi and LTE-LAA coexistence. IEEE Transactions on Cognitive Communications and Networking, 4(4), 735-748. https://doi.org/10.1109/TCCN.2018.2867032

[13] Wang, X., Quek, T. Q., Sheng, M., \& Li, J. (2016). Throughput and fairness analysis of Wi-Fi and LTE-U in unlicensed band. IEEE Journal on Selected Areas in Communications, 35(1), 63-78. https://doi.org/10.1109/JSAC.2016.2632629

[14] Yuan, H., Guo, W., \& Wang, S. (2016). Device-to-device communications in LTE-unlicensed heterogeneous network. 2016 IEEE 17th international workshop on signal processing advances in wireless communications (SPAWC), 1-5. https://doi.org/10.1109/SPAWC.2016.7536872

[15] Wu, Y., Guo, W., Yuan, H., Li, L., Wang, S., Chu, X., \& Zhang, J. (2016). Device-to-device meets LTE-unlicensed. IEEE Communications Magazine, 54(5), 154-159. https://doi.org/10.1109/MCOM.2016.7470950

[16] Ismaiel, B., Abolhasan, M., Smith, D., Ni, W., \& Franklin, D. (2017). A survey and comparison of device-to-device architecture using LTE unlicensed band. 2017 IEEE 85th Vehicular Technology Conference (VTC Spring), 1-5. https://doi.org/10.1109/VTCSpring.2017.8108256

[17] Alkurd, R., Shubair, R. M., \& Abualhaol, I. (2014). Survey on device-to-device communications: Challenges and design issues. 2014 IEEE 12th International New Circuits and 
Systems Conference (NEWCAS), 361-364. https://doi.org/10.1109/NEWCAS.2014.6934057

[18] Ahmad, M., Naeem, M., Ahmed, A., Iqbal, M., \& Anpalagan, A. (2016). Mesh adaptive direct search approach for D2D resource management. Wireless Communications and Mobile Computing, 16(15), 2329-2339. https://doi.org/10.1002/wcm.2686

[19] Chen, B., Zheng, J., \& Zhang, Y. (2015). A time division scheduling resource allocation algorithm for D2D communication in cellular networks. 2015 IEEE International Conference on Communications (ICC), 54225428. https://doi.org/10.1109/ICC.2015.7249186

[20] Lee, J., Gu, J., Bae, S. J., \& Chung, M. Y. (2013, January). A resource allocation scheme for improving user fairness in device-to-device communication based on cellular networks. Proceedings of the 7th international conference on ubiquitous information management and communication, 16. https://doi.org/10.1145/2448556.2448668

[21] Liu, J., Shi, Y., Zhang, Y., Wang, X., Sun, H., \& Sheng, M. (2016). DO-Fast: a round-robin opportunistic scheduling protocol for device-to-device communications. Wireless Communications and Mobile Computing, 16(5), 519-537. https://doi.org/10.1002/wcm.2551

[22] Lin, X., Ratasuk, R., \& Ghosh, A. (2015). Network-assisted device-to-device scheduling in LTE. 2015 IEEE 81st Vehicular Technology Conference (VTC Spring), 1-5. https://doi.org/10.1109/VTCSpring.2015.7145948

[23] Zhang, H., Liao, Y., \& Song, L. (2017). D2D-U: Device-todevice communications in unlicensed bands for $5 \mathrm{G}$ system. IEEE Transactions on Wireless Communications, 16(6), 3507-3519. https://doi.org/10.1109/TWC.2017.2683479

[24] Liu, R., Yu, G., Qu, F., \& Zhang, Z. (2016). Device-todevice communications in unlicensed spectrum: Mode selection and resource allocation. IEEE Access, 4, 47204729. https://doi.org/10.1109/ACCESS.2016.2603237

[25] Xiao, Y., Chen, K. C., Yuen, C., \& DaSilva, L. A. (2014, April). Spectrum sharing for device-to-device communications in cellular networks: A game theoretic approach. 2014 IEEE International Symposium on Dynamic Spectrum Access Networks (DYSPAN), 60-71. https://doi.org/10.1109/DySPAN.2014.6817780

[26] AlAmmouri, A., Andrews, J. G., \& Baccelli, F. (2017). SINR and throughput of dense cellular networks with stretched exponential path loss. IEEE Transactions on Wireless Communications, 17(2), 1147-1160. https://doi.org/10.1109/TWC.2017.2776905

[27] Abramson, M. A., Audet, C., Dennis Jr, J. E., \& Digabel, S. L. (2009). OrthoMADS: A deterministic MADS instance with orthogonal directions. SIAM Journal on Optimization, 20(2), 948-966. https://doi.org/10.1137/080716980

\section{Contact information:}

\section{Chigang XING}

School of Information Engineering,

Wuhan University of Technology,

Wuhan University of Technology, Wuhan, 430070, China

E-mail: xingchigang@hotmail.com

\section{Fangmin LI}

(Corresponding author)

School of Computer Engineering and Applied Mathematics,

Changsha University,

No. 98, Hongshan Road, Kaifu District, Changsha, 410022, China

E-mail: Ifm@ccsu.edu.cn 